\title{
Investigation of Rotors Imitating Bird Wings to Reduce Electricity Consumption of HORNET
}

\author{
Yogo Takada ${ }^{\mathrm{a}, *}$, Tatsuki Kanada ${ }^{\mathrm{a}}$, Takahiro Tanaka ${ }^{\mathrm{a}}$ and Yodai Matsumura ${ }^{\mathrm{a}}$ \\ ${ }^{\text {a }}$ Osaka City University, Japan
}

\begin{abstract}
To address the deterioration of bridges and buildings, we have developed a robot, HORNET, to inspect building walls. This robot travels on the wall surface using propellers with wheels on the wall. As the running duration is short on smooth surfaces, the crosssectional shape of the rotor was designed to reduce the power consumption using two-dimensional numerical analysis using a genetic algorithm. Furthermore, we manufactured several kinds of rotors imitating birds and performed experiments to determine the ideal shape to reduce electricity consumption. Experiments were also conducted to evaluate the performance when the rotor was attached to HORNET. First, we developed HORNET's dynamic model and evaluated the performance by running a simulation on a route imitating the actual inspection route. Additionally, by installing a new rotor in HORNET, we evaluated the running performance on the up and down paths.
\end{abstract}

Index Terms - Robot, Bird Wings, Electricity Consumption, Cross-Sectional Shape, Wing Planform.

\section{INSTRUCTIONS}

The deterioration of bridges [1][2] and buildings [3] is a problem; hence, regular inspections are manually conducted. However, in addition to the enormous cost and time required, these inspections involve risks for the inspector. Therefore, in our laboratory, we have developed a robot, called HORNET [4], which moves along the wall of the structure so that inspection can be carried out cheaply and safely. For practical applications in inspection of walls, reduction of electricity consumption is required. The aerodynamic performance of the rotor is the main factor for optimization of the shape of the rotor. However, the Reynolds number of the flow around the rotor of HORNET is approximately several tens of thousands so that research of rotors in this application is not as advanced as that of wings in airplanes and helicopters. Therefore, to the reduce power consumption, we address the following aspects in three separate sections:

In section 2, we calculate the two-dimensional crosssection using a genetic algorithm (GA), which is a semioptimization method and is incorporated in the GTT code [5][6] for computation fluid dynamics (CFD) developed in this laboratory. Therefore, we obtain an improved cross-sectional shape for the rotor. In Section 3 , we study planar shapes of bird wings based on the range of Reynolds number for the application areas of HORNET. In section 4, we introduce a comparison of the performance of the rotor developed in this study using the dynamic model of HORNET. Finally, we evaluate whether reduction in electricity consumption by installing a new rotor in HORNET.

\section{CROSS-SECTIONAL SHAPE OF ROTOR}

We used a GA, which is commonly used for semioptimization of rotor cross-sectional shape. GAs are used as an optimization method to simulate genetic operations in the evolution process of organisms. When incorporating GA into the GTT code, it is necessary to parameterize the grid generation for the rotor crosssection. As the shape of the airfoil is designed to have the maximum camber, the maximum camber position, and the maximum blade thickness ratio, these parameters were set as the tuning parameters in the GA. Subsequently, the fitness function $\operatorname{val}(t)$, which shows how well the rotor adapts to the environment, is as shown in Eq. (1) was set. Here, $t$ is the generation number, $C_{D 0}$ and $C_{L 0}$ are the initial drag and lift coefficients of the airfoil, respectively. $C_{D}(t)$ and $C_{L}(t)$ are the drag and lift coefficients of each airfoil, respectively, in the generation number $t . C_{C D}$ and $C_{C L}$ represent weighting factors of the drag and lift, respectively. According to Eq. (1), the fitness $\operatorname{val}(t)$ becomes larger if the drag coefficient becomes smaller or if the lift coefficient becomes larger; however, the fitness val $(t)$ becomes smaller if the drag coefficient becomes larger or the lift coefficient becomes smaller. Therefore, it is desirable that the fitness $\operatorname{val}(t)$ is as large as possible.

$$
\operatorname{val}(t)=\frac{1}{C_{C D}\left[\frac{C_{D}(t)}{C_{D 0}}\right]+C_{C L}\left[\frac{C_{L 0}}{C_{L}(t)}\right]}
$$

After constructing the rotors, we conducted an experiment to investigate the performance of the rotor with the device as shown in Fig. 1 (a). In the device, since the moving part with the rotor is connected via the thrust bearings to the rods, it can float experiencing less friction as shown in Fig. 1 (b). The experimental method is described with reference to Fig. 2. First, the moving part is placed on a weight scale to measure the lift force. When the rotor is stationary, the mass of the moving part is displayed as $400 \mathrm{~g}$ on the weight scale. When the rotor 
is rotated in this state, lift is generated, and if the lift is $100 \mathrm{gf}(=0.981 \mathrm{~N})$, the weight scale displays as $300 \mathrm{~g}$ with the moving part remaining in contact with the weight scale. Therefore, using this device, the lift force can be measured. Although this device may affect the downwash from the rotor blades, the experiments in this study were conducted without consideration of this bad effect.

Simultaneously, rotational speed, voltage and electric current can also be measured. Power consumption can be obtained by measuring the voltage and current from a DC power supply. The voltage was set to $7.9 \mathrm{~V}$, and the current was measured with current sensor ACS712, Allegro MicroSystems LLC when the weight scale indicated to $185 \mathrm{~g}$.

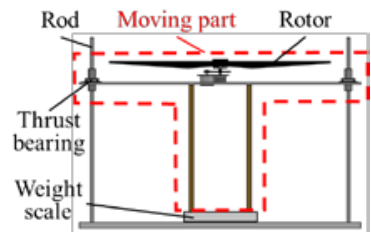

(a) Structure

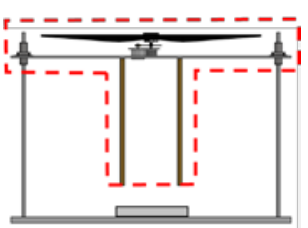

(b) Movement of the movable part

Fig. 1. Experimental device to investigate the performance of the rotor
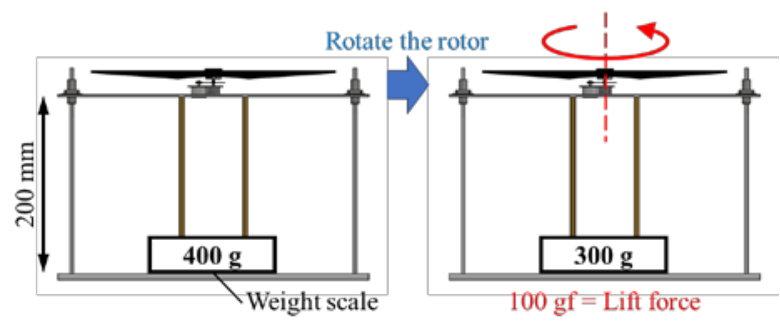

Fig. 2. Measurement method of lift

Table 1 and Fig. 3 show the performance and shapes, respectively, for the wing type MA409 (actual) [7] as a criterion for evaluation and for the new airfoils generated using numerical analysis incorporating GA. From Table 1 , it can be seen that the wing type GA15-9, which has the highest fitness, is a well-balanced airfoil with a larger lift coefficient and a sufficient lift drag ratio.

Table 1. Performance of MA409 and new airfoils

\begin{tabular}{c|c|c|c|c}
\hline \hline Airfoil & $C_{D}$ & $C_{L}$ & $C_{L} / C_{D}$ & $\operatorname{val}(t)$ \\
\hline MA409 (actual) & 0.0509 & 0.714 & 14.03 & 1.00 \\
\hline GA15-9 & 0.0965 & 1.168 & 12.10 & 1.35 \\
\hline GA15-5 & 0.1299 & 1.221 & 9.40 & 1.28 \\
\hline
\end{tabular}

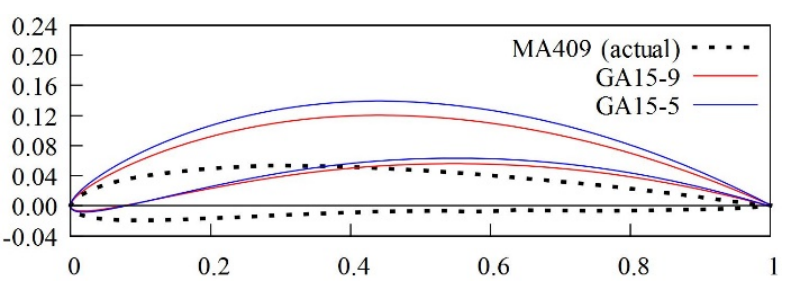

Fig. 3. Shapes of MA409 and new airfoils

Subsequently, we made a rotor with an airfoil crosssectional shape calculated using the aforementioned analysis using a 3D printer, and conducted an experiment using this rotor. The results are shown in Fig. 4. In Fig. 4 the relationship between the required power for hovering and fitness is shown. As the fitness increases, it can be seen that the power consumption is reduced. This result indicates that GA15-9 made by a 3D printer superior like the result of CFD analysis. Compared to MA409 (actual), GA15-9 can reduce the power consumption by approximately $18.8 \%$. Therefore, we limit our study to the GA15-9.

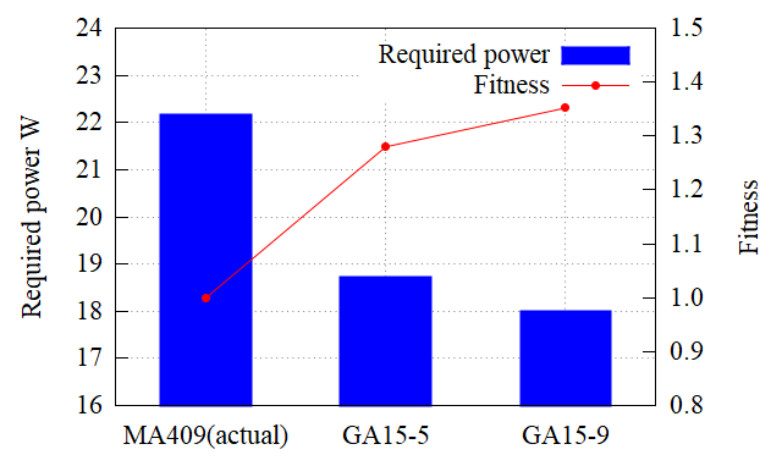

Fig. 4. Relationships between power required for hovering and fitness

\section{PLANAR SHAPE OF THE ROTOR}

In investigating the planar shape of the rotor, we imitated the wings of birds. Although bird-wings have various shapes, the wings can be divided into sharpedged wings and wings with wing-tip feathers. The sharp-edged wings have features such as reducing the drag by minimizing the turbulence generated at the tip of the wing. Thus, they are suitable for high speed flight. Wings with wing-tip feathers have features such as diffusing the turbulence generated at the tip of the wing to reduce drag; thus, making them suitable for low-speed flight [8]. In this study, planar rotors that imitated the sharp-edged wing of a bird have been manufactured, as shown in Fig. 5.

The shape $\mathrm{A}$ is the same as that used in the experiment to obtain the cross-sectional shape GA15-9 of the rotor in the previous section. The shapes $\mathrm{B}$ and $\mathrm{C}$ were manufactured based on sharp-edged wings especially like an albatross. In addition to sharpening the rotor blade tip shape, the rotor blade of shape $C$ was manufactured based on a bird's wing shape by attaching a forward angle near the blade root. The results of experiments using these rotors are shown in Fig. 6.

As the evaluation method of the rotor based on elementary blade element theory was applied. Given that the inflow velocity of air changes depending on the distance from the center of rotation of the rotor, the force acting on each length element (blade element) of the rotor at the radius from the center of rotation is calculated separately. Elementary blade element theory was used to obtain the characteristics of the whole rotor from the force applied to each separate rotor blade element. Furthermore, we assume that there is no interference between adjacent blade elements and that the force 
acting on the blade element is only due to the lift coefficient and the drag coefficient of the cross-sectional shape of the blade. The results based on the analysis using this theory are shown in Fig. 7, where parameter $x$ shows the relative position in Fig. 5.

According to the result shown in Fig. 6, it can be seen that the required power for shape $\mathrm{C}$ is the smallest. Subsequently, the vertical axis in Fig. 7 shows dimensionless lift coefficients which are divided by the product of the dynamic pressure and the average chord. The lift coefficients in the vicinity of the blade tip in shapes B and C are smaller than that of shape A because their wing tips are sharp. However, the lift coefficient of shape $C$ is larger than that of shape A except for the wing tip. Besides, from the same figure, in case of shape $C$, the lift coefficients are larger on a position far from the rotation center as compared with shape B whose lift coefficients are large near the rotation center. The required electric power for hovering (185 g per one rotor) can be suppressed because the shape $\mathrm{C}$ may obtain a large lift at even a slow peripheral velocity.

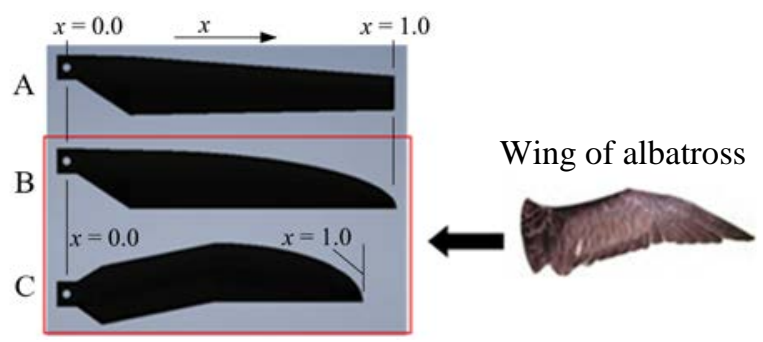

Fig. 5. Top view of each rotor

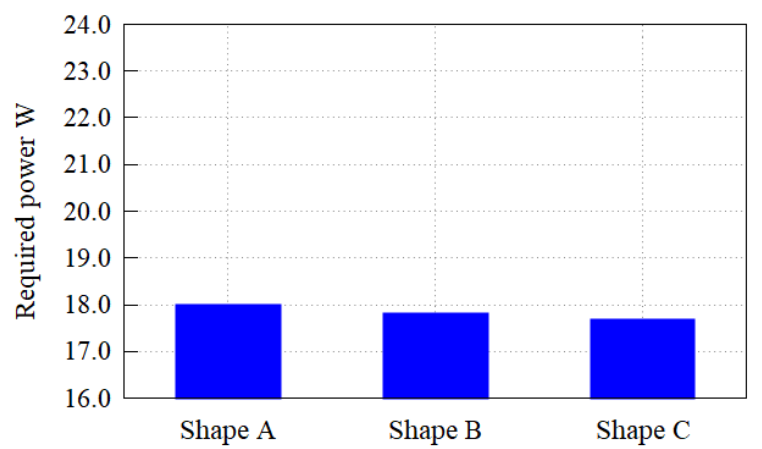

Fig. 6. Relationship between planar shape and power required for hovering

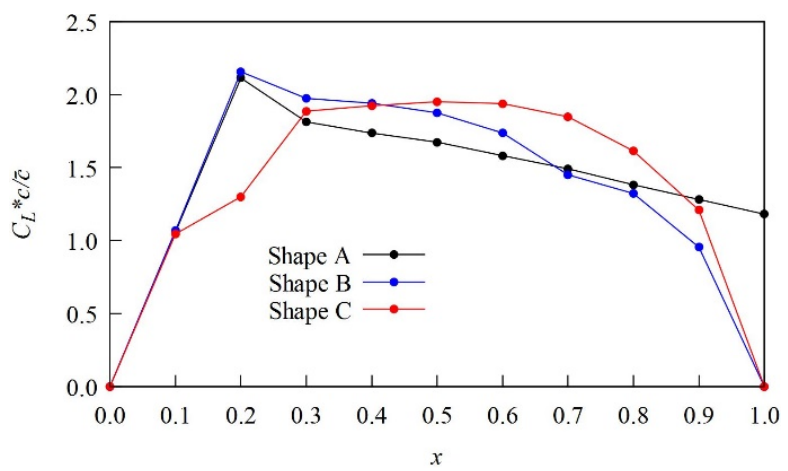

Fig. 7. Relationship between the planar shape and spanwise lift distribution
When a bird descends in flight, the angle of attack of the outer wing is decreased, thus suppressing the generation of the opposite force [9]. The robot HORNET in this study rotates the rotor, unlike birds. However, the movement of the tip of the wing is faster than those closer to the body when birds descend in flight. Furthermore, the outer side of rotor is much faster than inner side of the rotor. Therefore, we believe that the characteristic of the rotor are similar to the bird wing. Therefore, we manufactured the twisted rotors (as shown Fig. 8) for HORNET by imitating the feathering that twists the wing so that the leading edge of the wing faces downward when swing the wing down.

Regarding the twist-down, the blades of a helicopter are generally provided with a linear twist-down of $8^{\circ}$. In the case of bird wings, there is a weak twist down from the body to the vicinity of the center of the wing. Additionally, a strong twist-down exists from the center to the tip of the wing. Therefore, shapes A2 and C2 were completely linearly twisted-down, compared to shapes A and $\mathrm{C}$ rotors. Here, the twist-down angle was set at $8^{\circ}$. Subsequently, for shape C3, the rotor blade was twisted by $8^{\circ}$ only between the middle of the wing (vertical red line on shape $C$ in Fig. 8) and the tip of the wing was similar to that of a bird wing.

The experimental results are shown in Fig. 9, and the analytical results using elementary blade element theory are shown in Fig. 10. According to Fig. 9, both shapes A and $\mathrm{C}$ show better performance when the blade twisted down. In shapes $\mathrm{A}$ and $\mathrm{C}$, shape $\mathrm{C}$ performs better. However, from the result of the comparison between twisted-down shapes A2 and C2, A2 outperformed better than C2. Furthermore, as with the wing twisting-down of birds, shape C3 had the best performance. Subsequently, according to Fig. 10, it was confirmed that the performance improves when the lift distribution is nearly constant such as shapes A2 and C3. If the lift distribution is not constant, high and low air pressure areas are formed, and a stall will occur. As a result, aerodynamic performance will deteriorate. However, as the lift distribution of the shapes A2 and C3 is almost constant, a high-pressure region and a low-pressure region are formed little and we considered that a stall has decreased. Furthermore, by comparing shapes A2 and C3, it was confirmed that the performance improves when the lift is small near the blade tip. In shape C3,

A2

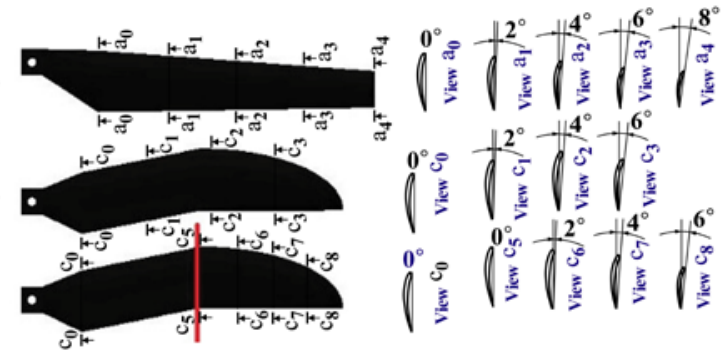

Fig. 8. Top view of rotors (A2 and C2) with $8^{\circ}$ twist throughout, and a rotor (C3) with $8^{\circ}$ twist between a red line and the tip 
the lift does not decrease at $x=0.9$, compared to shape $\mathrm{C} 2$, and the lift distribution is found to be nearly constant.

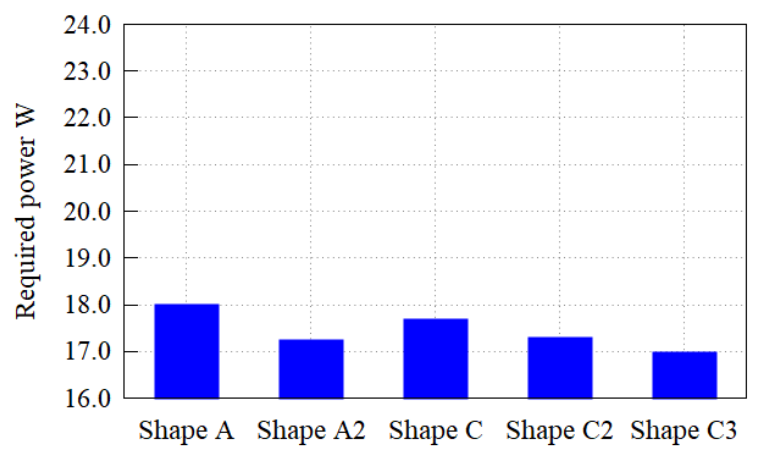

Fig. 9. Relationship between the power required for hovering and the difference in twist-down shapes.

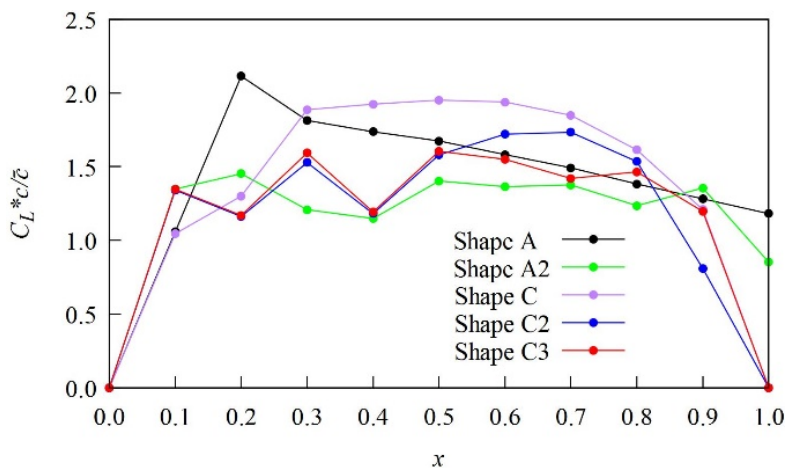

Fig. 10. Relationship between the spanwise lift distribution and the difference in twist-down shapes.

\section{EVALUATION OF ROTOR}

HORNET is a robot that can move vertically on walls with ease. Figure 11 shows a photograph of HORNET and Table 2 shows HORNET specifications. HORNET has two rotors, brushless DC motors for rotating them, a servomotor (TS-1002 made by Tahmazo) for changing the tilt angle of the rotor surface, two wheels with claws on the outer circumference, an electronic circuit for control, a receiver for receiving signals from external transmitters, motor drivers for controlling the right and left brushless DC motors, a lithium polymer battery, and a metal ball for the hammer test for a wall. Zynq (TE0720-03-1CF, manufactured by Xilinx Inc.) is used

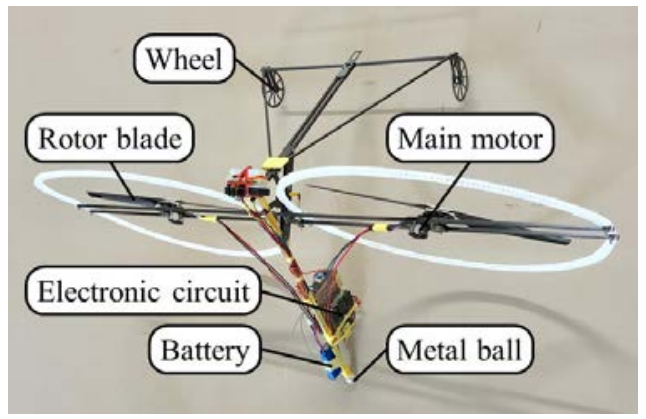

Fig. 11. Photograph of HORNET
Table 2. Specifications of HORNET

\begin{tabular}{c|c}
\hline \hline Weight & $310 \mathrm{~g}$ \\
\hline Size & $350 \mathrm{~mm} \times 650 \mathrm{~mm} \times 350 \mathrm{~mm}$ \\
\hline Turning diameter of rotor & $300 \mathrm{~mm}$ \\
\hline Battery & $\begin{array}{c}\text { Lithium polymer battery, } \\
7.4 \mathrm{~V}, 600 \mathrm{mAh}, 30 \mathrm{C}\end{array}$ \\
\hline Control IC & $\begin{array}{c}\text { Zynq (TE0720-03-1CF, } \\
\text { manufactured by Xilinx) }\end{array}$ \\
\hline Receiver & $\begin{array}{c}\text { R2006GS } \\
\text { (manufactured by Futaba) }\end{array}$ \\
\hline
\end{tabular}

as the controller. The signal sent from the transmitter, which has the joysticks for controlling HORNET, is received by the FPGA via the receiver. Furthermore, the FPGA controls the rotational speed of the right and left propeller driving motors and the tilt angle of the rotor surface.

Evaluation of the performance of rotor C3 is discussed in Section 3 is conducted for its installation on HORNET. The evaluation metrics were the duration and the distance that HORNET was able to move during a single battery discharge. However, given that HORNET cannot run autonomously on a route that requires horizontal movements, it is difficult to evaluate the performance of the rotor traveling on the route shown in Fig. 12. Therefore, we constructed a model of HORNET and evaluated the rotor via a computer simulation using the model.

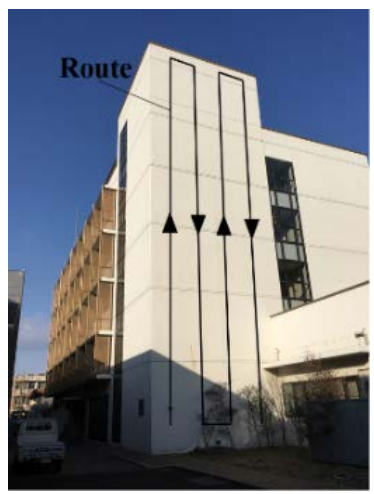

(a) Building $\mathrm{G}$ at O.C.U.

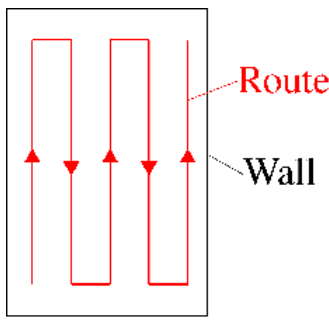

(b) Simulation route
Fig. 12. Route of HORNET

The equation of motion for HORNET is expressed as Eq. (2). This robot has eight degrees of freedom, including rotation of the left and right rotors.

$$
\boldsymbol{M}(\ddot{\boldsymbol{q}})+\boldsymbol{h}(\dot{\boldsymbol{q}}, \boldsymbol{q})+\boldsymbol{g}(\boldsymbol{q})=\boldsymbol{Q}
$$

In Eq. (2), $\boldsymbol{M}$ represents mass and moment of inertia, $\boldsymbol{h}$ represents centrifugal force and Coriolis force, and $\boldsymbol{g}$ represents gravity.

$$
\begin{gathered}
\boldsymbol{q}=\left(\begin{array}{llllllll}
x_{c} & y_{c} & z_{c} & \alpha & \beta & \gamma & \theta_{R} & \theta_{L}
\end{array}\right)^{T} \\
\boldsymbol{Q}=\left(\begin{array}{llllllll}
F_{x} & F_{y} & F_{z} & \tau_{\alpha} & \tau_{\beta} & \tau_{\gamma} & \tau_{\theta_{R}} & \tau_{\theta_{L}}
\end{array}\right)^{T}
\end{gathered}
$$

The vector $\boldsymbol{q}$ of Eq. (3) has the state variable expressed by the position of the robot $\left(\begin{array}{lll}x_{c} & y_{c} & z_{c}\end{array}\right)^{T}$, $\left(\begin{array}{lll}\alpha & \beta & \gamma\end{array}\right)^{T}$ and the left and right rotor rotation 
angle $\left(\theta_{R} \quad \theta_{L}\right)^{T}$. Here, $\alpha, \beta, \gamma$ are ZYX Euler angles. The vector $\boldsymbol{Q}$ of Eq. (4) is subjected to the forces $F_{x}, F_{y}$, and $F_{z}$ externally, the torques $\tau_{\alpha}, \tau_{\beta}$, and $\tau_{\gamma}$ are applied to each axis direction using these external forces, and the left and right motor shaft torques $\tau_{\theta_{R}}, \tau_{\theta_{L}}$. The force and torque of Eq. (4) correspond to the state variables of Eq. (3). After converting the obtained second-order ordinary differential equations having eight unknown coefficients to a first-order ordinary differential equations with 16 unknown coefficients, numerical calculation was performed using the RungeKutta method.

For example, HORNET moves vertically and horizontally along a wall of a building as shown in Fig. 12 (a). Therefore, HORNET was simply moved as shown in Fig. 12 (b), even in the simulation. Specifically, operations such as moving $2 \mathrm{~m}$ upwards, $0.2 \mathrm{~m}$ to the right, $2 \mathrm{~m}$ downwards, and $0.2 \mathrm{~m}$ to the right, are repeated. The simulation results are shown in Tables 3 and 4 . Table 3 shows the simulation results until the battery voltage falls below the rated voltage. From Table 3 , it can be seen that duration of functioning for HORNET has increased from 6.1 minutes to 6.6 minutes. Furthermore, the moving distance also became $8.2 \%$ longer by changing to the rotor $\mathrm{C} 3$. In the case of the rotor $\mathrm{A}$, it was only possible to make 16 round trips with a single battery charge. However, the robot equipped with rotor C3 can make 18 round trips. Therefore, for example, when 50 round trips are necessary, three battery charges are required in rotor A, whereas in C3, it is possible to reduce the number to only two, as shown in Table 4.

Table 3. Results of running simulation on the route shown in Fig. 12 (b)

\begin{tabular}{c|c|c}
\hline \hline & A & C3 \\
\hline Duration (min) & 6.1 & 6.6 \\
\hline Moving distance (m) & 73.2 & 79.2 \\
\hline Round-trip numbers & 16 & 18 \\
\hline
\end{tabular}

Table 4. Battery exchange during the 50 round trips

\begin{tabular}{c|c|c|c|c|c|c|c}
\hline \hline Rotor A & 16 & $\mathrm{e}^{*}$ & 16 & $\mathrm{e}^{*}$ & 16 & $\mathrm{e}^{*}$ & 2 \\
\hline Rotor C3 & 18 & $\mathrm{e}^{*}$ & 18 & $\mathrm{e}^{*}$ & 14 & : \\
\hline \multicolumn{10}{c}{ means a battery exchange. }
\end{tabular}

The total lift force of HORNET is expressed by $F_{z}$ in Eq. (4). It is the sum of the lift produced by the left and right Rotor blades. The lift produced by the left or right rotor blade at a stational state of HORNET on a wall is shown in Fig. 13 and it depends on the rotational speed. Besides, the motor torque required to maintain the rotational speed is shown in Fig. 14.

The rotor blades of HORNET rotate at around 2500 rpm to support the weight of the main body at a stational state on the wall. Fig. 13 shows that each lift of Shape A and Shape $\mathrm{C}$ at the rotational speed is almost the same. However, the required motor torque to rotate at the rotational speed is less for Shape C than for Shape B.

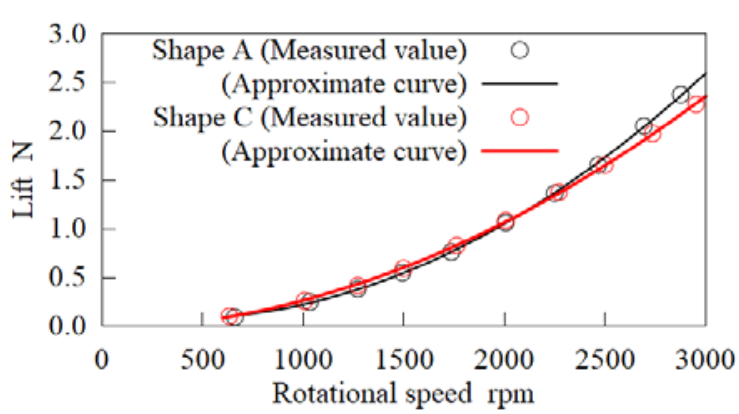

Fig. 13. Relationship between lift and rotational speed about Shape A and Shape C (Measured values are obtained by the experimental device of Figs.1 and 2)

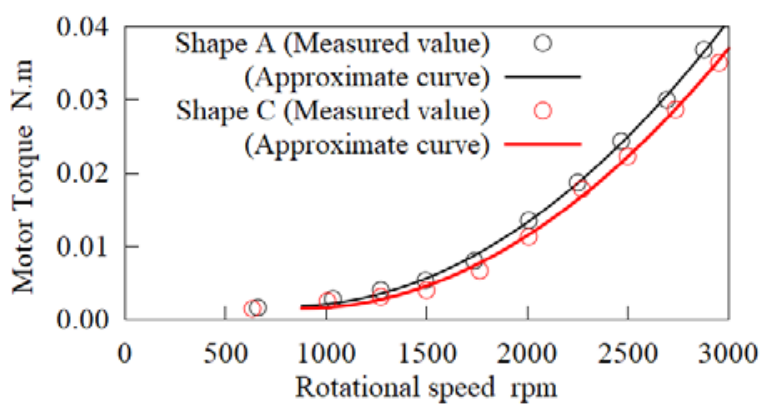

Fig. 14. Relationship between the motor torque and rotational speed about Shape A and Shape C (measured values are calculated by measurement electric current)

We conducted similar experiments using HORNET robot and evaluated power consumption and moving distance. To measure the power consumption, a current sensor (module with ACS 711, Pololu) was attached to the robot. In addition, an ultrasonic sensor (US-015, SainSmart) and a six-axis acceleration and gyro sensor (KP-9250, Kyoritsu Electronics Industry Co., Ltd.) were attached to control the altitude of HORNET. Therefore, the mass of HORNET increased by $56 \mathrm{~g}$ to $366 \mathrm{~g}$. In this experiment, HORNET was simply moved vertically as shown in Fig. 15. The operations such as moving $1.2 \mathrm{~m}$ upwards and $1.2 \mathrm{~m}$ downwards, are repeated until the battery voltage become lower than the rated voltage or became unable to generate the lift required for moving upward. The result of the experiment and the average electric power consumption are shown in Table 5 and Fig. 16. Table 5 shows that application of the C3 rotor has reduced the average electric power consumption by $9.0 \%$ and has increased the movement range of HORNET by $7.1 \%$.

We considered the reason why the average electric power consumption of shape $\mathrm{C}$ decreased. The range from 2000 to $2750 \mathrm{rpm}$ of the rotation speed is used for moving up and down on the wall. According to the motor torque which is proportional to the current in Fig. 14, the load for rotating the shape $C$ rotor blade is as less as the rate of the average electric power consumption, and as a result, the drag of the shape $\mathrm{C}$ is suppressed. 


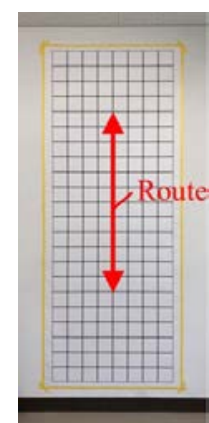

Fig. 15. Indoor wall used for the running experiment on which imitation paper is affixed

Table 5. Result of the experiment running only in the vertical direction

\begin{tabular}{c|c|c}
\hline \hline & A & C3 \\
\hline Duration (min) & 3.26 & 3.50 \\
\hline Moving distance (m) & 39.2 & 42.0 \\
\hline Round-trip numbers & 16 & 17 \\
\hline Power consumption (Wh) & 2.60 & 2.56 \\
\hline Average power (W) & 47.9 & 43.6 \\
\hline
\end{tabular}

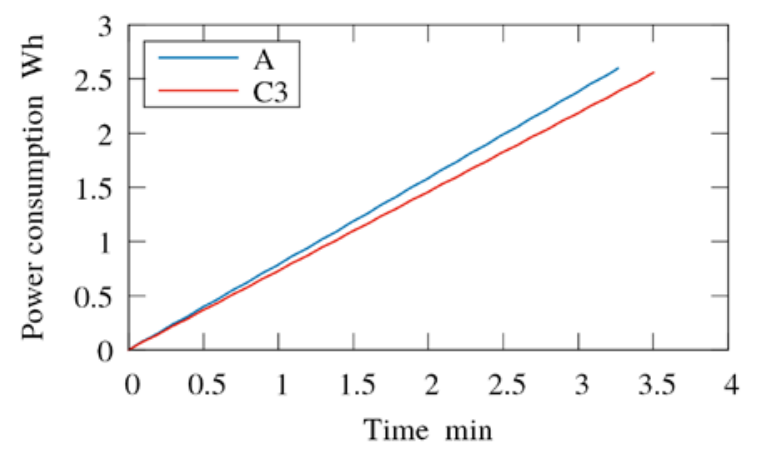

Fig. 16. Power consumption in the running experiment

\section{CONCLUSIONS}

In this study, various types of rotors for HORNET were developed with the goal of a practical application for a wall inspection robot.

In the low Reynolds number region of about several tens of thousands corresponding to the flow around the rotor of HORNET. CFD analysis was conducted on a two-dimensional cross-section incorporating a genetic algorithm (GA). Based on the cross-sectional shape obtained by the GA, we manufactured various types of rotors, and performed experiments to confirm the performance. Therefore, we confirmed that the rotor with a larger fitness function in GA had a better performance that the power required to keep HORNET stationary became small. As a result, we were able to develop a cross-section for the rotor with good performance for HORNET.

Using elementary blade element theory, we confirmed that rotor for which the lift near the blade tip was small and the overall lift distribution was constant tends to have lower power consumption in the low Reynolds number range. Given that bird wings meet this requirement, it was confirmed that the power consumption owing to the planar shape of the rotor can be reduced by imitating the wings of the bird.

We compared the performance of HORNET installing the rotor with different planar shape. First, we created a dynamic model of HORNET and evaluated its performance as it traveled a route that include horizontal movement by simulation. In addition, we evaluated the performance when moving up and down using the actual HORNET robot. In both experiments, the new rotor reduced the power consumption of HORNET. Therefore, by installing a the newly developed rotor, HORNET was able to function for longer on a single charge.

We have confirmed the performance improvement by adopting a bird wing shape and the effect of GA in this study. In the future, we will conduct CFD analysis on a three-dimensional space including two rotating rotor blades, and consider a method to improve the shape of the bird wing by using GA.

\section{ACKNOWLEDGEMENT}

A part of this study was supported by Grant-in-Aid for Scientific Research (C) 15K05905. The authors would like to express their sincere gratitude for the support.

\section{REFERENCES}

[1] T. Tamakoshi, M. Shirato, and T. Kamada, "Steel bridge deterioration data in Japan and modelling," Proc. of the Institution of Civil Engineers - Bridge Eng., Vol. 170, No. 2, pp. 133-146, June 2017.

[2] F. Ghodoosi, A. Bagchi, and T. Zayed, "System-Level Deterioration Model for Reinforced Concrete Brid Decks,” J. Bridge Eng., Vol. 20, No. 5, 04014081, 2015.

[3] C. Chen "A Study on the Deterioration External Wall Tiles on RC Buildings in Taiwan,” Applied Mechanics and Materials, ISSN: 1662-7482, Vols. 479-480, pp. 1105-1108, December 2013.

[4] Y. Tokura, K. Toba, and Y. Takada "Practical Applications of HORNET to Inspect Walls of Structures," Journal of Robotics and Mechatronics, Vol. 28, No. 3, pp. 320-327, June 2016.

[5] Y. Takada, Y. Nakanishi, R. Araki, M. Nonogaki, T. Wakisaka, "Effect of Material and Thickness about Tail Fins on Propulsive Performance of a Small Fish Robot," Journal of Aero Aqua Bio-mechanisms, Vol. 1, No. 1, pp. 51-56, 2010.

[6] Y. Takada, T. Ochiai, N. Fukuzaki, T. Tajiri, T. Wakisaka, "Analysis of Flow around Robotic Fish by Threedimensional Fluid-structure Interaction Simulation and Evaluation of Propulsive Performance,” Journal of Aero Aqua Bio-mechanisms, Vol. 3, No. 1, pp. 57-64, 2013.

[7] G. A. Williamson, B. D. Mc Granahan, B. A. Broughton, R. W. Deters, J. B. Brandt, and M. S. Selig, Summary of LowSpeed Airfoil Data, Vol. 5, pp. 313-339, 2012.

[8] N. A. Siddiqui, W. Asrar, E. Sulaeman, "Literature Review: Biomimetic and Conventional Aircraft Wing Tips," International Journal of Aviation, Aeronautics, and Aerospace, Vol. 4, Issue 2, Article 6, 2017.

[9] H. Ohtake, "Robotics on Bird-like Flapping Flight," Journal of the Robotics Society of Japan, Vol. 34, No. 1, pp. 14-18, 2016 (in Japanese). 